\title{
Recent trends on convergence and ubiquitous computing
}

\author{
Kyung-Yong Chung
}

Published online: 2 November 2013

(C) Springer-Verlag London 2013

Convergence of content and technologies is a theme that increasingly dominates the digital world. Applications, devices, networks, and infrastructure are converging and creating an immense amount of content, which poses complex challenges for organizations, developers, and users. In the case of ubiquitous computing, we are seeking the convergence of frameworks and paradigms such as augmented reality, ubiquitous computing, and pervasive computing. We are also seeing new kinds of content, much of it user-generated, that can be searched, organized, and consumed on many devices and in many formats. Challenges regarding converged technologies and content require new algorithms, application paradigms, interaction methods, and personalization services; creation of expressive annotation frameworks, scalable algorithms, and new paradigms for information discovery; and ways of searching, organizing, and delivering converged information for delivery at the right place, right time, and with the right level of detail [1-7].

- Convergence networks

- Converged frameworks

- Interactive multimedia applications

- Indexing, searching, and visualization

- Ontology-based multimedia information visualization

- Convergence in ubiquitous computing

- Innovative applications of ubiquitous computing

- Intelligent applications for multimedia processing

- Speech interfaces for ubiquitous computing

- User experience in converged systems

K.-Y. Chung ( $\square)$

School of Computer Information Engineering, Sangji University,

Wonju, Republic of Korea

e-mail: dragonhci@hanmail.net
- Personalization for ubiquitous healthcare

- Information indexing, searching, and visualization

- Intelligent information processing algorithms

- Sensors, wireless technology, and embedded systems

This theme issue has become one of the hottest topics in convergence and ubiquitous computing. We believe that this theme issue will have a high citation in the areas of personal and ubiquitous computing.

The first paper, by $\mathrm{Oh}$ et al., presents a robust vocabulary recognition-clustering model using an average estimator least-mean-square filter in a noisy environment. Their method allows the cancelation of noise with an average estimator least-mean-square filter in a noisy environment. A robust speech recognition-clustering model can be established. Using the clustering structure, it becomes possible to reduce the number of parameters that would otherwise increase, which expands the efficiency of modeling training. To measure the intensity of noise in an environment, the signal-to-noise ratio for each environment was calculated and used for performance evaluation. Recognition performance was evaluated by composing a Gaussian clustering model, which is a robust speech recognition-clustering model, in a noisy environment.

The second paper, by Choi et al., investigated the parallel efficiency of a PC cluster to simulate a CFD problem. This paper describes the configuration of the affordable PC hardware cluster as well as parallel computing performance using a commercial CFD code in the developed cluster. This cluster was designed to solve large fluid dynamics and heat transfer problems in parallel. Using a commercial CFD package, the performance of the cluster was evaluated by changing the number of CPU cores involved in the computation. A forced convection problem around a linear cascade was solved using the CFX program, and the heat 
transfer coefficient along the surface of the turbine cascade was simulated. To compare the experimental data, a numerical simulation model was designed with geometries and boundary conditions. The simulation results are drawn in a Nusselt number contour plot. The results were compared with the experimental Nusselt number at multiple locations.

The third paper, by Lim et al., presents the feature extraction using Hilbert transforms, phase-space reconstruction, and time-domain analysis to detect ventricular fibrillation and normal sinus rhythms from ECG episodes. For this purpose, they implemented three preprocessing steps to extract features from ECG episodes. First, using statistical methods, including frequency distributions and peak variability, they extracted the mean of the absolute peaks as well as the median, average power, and standard deviation. Second, using Euclidean distances based on PSR, they extracted the mean, median, average power, and standard deviation of the Euclidean distances. Using NEWFM, they obtained the BSWFM for 11 minimum features and to find fuzzy membership functions for the 11 features.

The fourth paper, by Park et al., presents a u-Silvercare service system using a LTE-A-based relay antenna to the solve problems in the heterogeneous network. For this system, they designed a square loop antenna with a frequency of $900 \mathrm{MHz}$, which is domestic SK's LTE band. In the proposed system, a multihop relay was applied in order to improve reception performance and solve the problem related to LTE system transmission, which is an OFDM method that uses a multicarrier with a large PAPR that causes a lot of noise in the signal when the AP and terminal are far apart. To maximize relay efficiency, relay power allocation and relay routing method were proposed. Selective transmission method was applied to improve reception of the LTE system. Collected experimental values and a previous system were compared and analyzed. Relatively low error rates of 0.21 and $1.53 \%$ were determined. Reception rates were better than those of the previous system.

The fifth paper, by Lee et al., identified the abnormal status of a patient using an acceleration algorithm. They proposed remote health-monitoring services targeting highrisk patients as well as elderly persons who live alone and require ongoing health checks. In the service, medical staff in remote locations is notified of any anomalies in the health of a patient. It is important to determine whether any abnormal symptom has occurred. According to the experimental results, temporary phenomena caused by sudden movement of the patient along with error factors caused by the temperature of the surrounding environment could be reduced. However, a disadvantage of applying these methods is that data must be collected previously for a period of time. That is, the system proposed in this research shows the same performance as the existing simple threshold method in the beginning while consuming more system resources.

The sixth paper, by Lee et al., presents an adaptive network-based fuzzy inference system along with linear discriminant analysis on the basis of a Korean national health and nutrition examination survey. The proposed coronary heart disease optimization system combines a fuzzy inference method and LDA to predict CHD, thus increasing the specificity, sensitivity, and accuracy of the model. In order to increase prediction accuracy, 625 rules were created using the sample medical data. Further, the classification performance was improved using an adaptive-network-based fuzzy inference system and linear discriminant analysis. The proposed method, which was optimized using a hybrid method, exhibits a high prediction rate of $80.2 \%$ and is more efficient and effective than previous methods.

The seventh paper, by Chung et al., presents an automotive design paradigm using relevance feedback. The developing system is an automotive design recommendation that increases the efficiency of merchandizing for design products and develops a recommendation system and user interface for an automotive design to satisfy user's needs. An automotive design paradigm prediction system that applies image-based collaborative filtering, which combines both image filtering and collaborative filtering, was implemented. The desired design could be recommended because image-based collaborative filtering plays a role in predicting automotive designs using image filtering and collaborative filtering. The process extracts features from image data in which users are interested in order to mitigate the problem, which is regarded as an undulated analysis, and then recommends an automotive design by combining image filtering and collaborative filtering, which uses the correlation relationship between the preference and image information. The recommendation according to sensibility and tendency is made possible by applying the relevance feedback to react to the paradigm, which changes according to various lifestyles.

The eighth paper, by Park et al., presents a dangerous Wi-Fi access point: attacks to benign smartphone applications. They attempted to reveal the risk of using unknown APs by presenting demonstration results. The test bed was composed of two smartphones, two APs, and one server. The compromised AP forwards messages from the victim's smartphone to the fake server using DNS spoofing. Thus, the application running on the victim's smartphone transfers the HTTP request to the fake server. As a result, test applications that are launched on the handset display abnormal advertisements. They showed that benign applications running on uncompromised devices can be 
exploited just connecting to the compromised AP. To mitigate this MITM attack, the developer must use mutual authentication processes when an application communicates with external devices.

The ninth paper, by Rim et al., presents a multiple categorization of products: cognitive modeling of customers through social media data mining. In this study, the authors followed the concept of a constructed market and argue that objective information is required for decision-making, while preferences and sentiment subjectively play important roles in product innovation and purchases. Previous studies tend to concentrate on indirect observations of the flow of information or resources through inter-actor connections or longitudinal measurements of a single experiment or a survey. Further, they suggested the possibility of structuring the formation of public opinion and the diffusion network by observing the internal dynamics of online communities from the perspectives of human-computer interaction, management information systems, and digital marketing.

The tenth paper, by Park et al., presents a cross-layer approach for multiuser multiplexing on IP mobility. They proposed service quality improvement methods by acquiring multiuser channel state information for multicast video streaming transmission using a method implemented between network layers. The channel state information of each user in the multicast group is used as information for the transmission of multicast packets. They also propose optimization methods of a cross-layered approach for wireless network multimedia communication systems and video streaming application services. The proposed method between layers is based on sharing channel state information from the physical layer in a multicast packet scheduler. As a result of the simulation, video streaming service performance for multicast users was improved by applying the method between layers.

The eleventh paper, by Jung et al., introduces an extracting communication structure of a development organization from a software repository. They suggested a way to extract the development organization from software repositories with respect to the temporal locality. In this system, they used two metrics: NoC and CF. NoC is based on the number of commits a developer has made to the system and measures how much the developer has contributed to development. For this purpose, they built a tool, ArchOrg, which can automatically extract the communication structure as a graph that has $\mathrm{NoC}$ as the size of the node and CF the as distance between nodes. They validated our approach with empirical data from the Eclipse JDT project.

The last paper, by Lee et al., presents the impact of policy measures on promoting the modal shift from road to rail. They first formulate hypotheses that describe the effects between policy measures and modal shift. For the purpose, they verified this impact with statistical analyses through testing hypotheses, which are established from findings in previous research. Second, they developed a model describing effects between policy measures and clarified their directivity with structural equation modeling. As a result of the simulation, strategic planning and operations need to build appropriate infrastructure, which attracts freight transportation from road to rail. Services have a direct effect on strategic planning and operations as well as an indirect effect on infrastructure. Strategic planning and operations have a direct effect on infrastructure.

Acknowledgments This fine collection of papers was achieved by fruitful collaborations. We gratefully acknowledge and express heartfelt thanks to all the authors for their worthy contribution to this theme issue. We also would like to thank all the members of the committee and anonymous reviewers for their help in identifying the novel papers and for their careful reading of earlier drafts in order to select 12 high-quality papers out of 45 submitted papers, 26 percent acceptance rate. Furthermore, we would like to thank Professor Peter Thomas, editor-in-chief of the international research journals Personal and Ubiquitous Computing (PUC), for his valuable remarks and his undeterred help throughout the publication process of this theme issue.

\section{References}

1. Weiser M (1993) Ubiquitous computing. J Comput 26(10):71-72

2. Kim SH, Chung KY (2013) Medical information service system based on human 3D anatomical model. Multimed Tools Appl. doi:10.1007/s11042-013-1584-8

3. Kim JH, Ryu JK (2013) Recent trends on high-performance computing and security. Cluster Comput 16:207-208

4. Oh SY, Chung KY (2013) Target speech feature extraction using non-parametric correlation coefficient. Cluster Comput. doi:10. 1007/s10586-013-0284-5

5. Chung KY, Na YJ, Lee JH (2013) Interactive design recommendation using sensor based smart wear and weather webbot. Wirel Pers Commun 73(2):243-256

6. Jung EY, Kim JH, Chung KY, Park DK (2013) Home health gateway based healthcare services through u-health platform. Wirel Pers Commun 73(2):207-218

7. Baek SJ, Han JS, Chung KY (2013) Dynamic reconfiguration based on goal-scenario by adaptation strategy. Wirel Pers Commun 73(2):309-318 